\title{
FIXED POINT ITERATION FOR ASYMPTOTICALLY \\ QUASI-NONEXPANSIVE MAPPINGS \\ IN BANACH SPACES
}

SOMYOT PLUBTIENG AND RABIAN WANGKEEREE

Received 21 October 2004 and in revised form 20 April 2005

Suppose that $C$ is a nonempty closed convex subset of a real uniformly convex Banach space $X$. Let $T: C \rightarrow C$ be an asymptotically quasi-nonexpansive mapping. In this paper, we introduce the three-step iterative scheme for such map with error members. Moreover, we prove that if $T$ is uniformly $L$-Lipschitzian and completely continuous, then the iterative scheme converges strongly to some fixed point of $T$.

\section{Introduction}

Let $C$ be a subset of normed space $X$, and let $T$ be a self-mapping on $C$. $T$ is said to be nonexpansive provided that $\|T x-T y\| \leq\|x-y\|$ for all $x, y \in C$; $T$ is called asymptotically nonexpansive if there exists a sequence $\left\{k_{n}\right\}$ in $[0, \infty)$ with $\lim _{n \rightarrow \infty} k_{n}=0$ such that $\left\|T^{n} x-T^{n} y\right\| \leq\left(1+k_{n}\right)\|x-y\|$ for all $x, y \in C$ and $n \geq 1$. $T$ is said to be an asymptotically quasi-nonexpansive map if there exists a sequence $\left\{k_{n}\right\}$ in $[0, \infty)$ with $\lim _{n \rightarrow \infty} k_{n}=0$ such that $\left\|T^{n} x-p\right\| \leq\left(1+k_{n}\right)\|x-p\|$ for all $x \in C$ and $p \in F(T)$, and $n \geq 1(F(T)$ denotes the set of fixed points of $T$, that is, $F(T)=\{x \in C: T x=x\})$.

From the above definitions, if $F(T) \neq \varnothing$, then asymptotically nonexpansive mapping must be asymptotically quasi-nonexpansive mapping.

The concept of asymptotically nonexpansiveness was introduced by Goebel and Kirk in 1972 [2]. In 2001, Noor [5, 6] introduced the three-step iterative scheme and he studied the approximate solutions of variational inclusions (inequalities) in Hilbert spaces. The three-step iterative approximation problems were studied extensively by Noor $[5,6]$, Glowinski and Le Tallec [1], and Haubruge et al. [3].

Recently, $\mathrm{Xu}$ and Noor [8] introduced the three-step iterative scheme for asymptotically nonexpansive mappings and they proved the following strong convergence theorem in Banach spaces.

Theorem 1.1 (see [8, Theorem 2.1]). Let X be a real uniformly convex Banach space, let $C$ be a nonempty closed, bounded convex subset of $X$. Let $T$ be a completely continuous and asymptotically nonexpansive self-mapping with sequence $\left\{k_{n}\right\}$ satisfying $k_{n} \geq 0$ and 
$\sum_{n=1}^{\infty} k_{n}<\infty$. Let $\left\{\alpha_{n}\right\},\left\{\beta_{n}\right\}$, and $\left\{\gamma_{n}\right\}$ be real sequences in $[0,1]$ satisfying

(i) $0<\liminf _{n \rightarrow \infty} \alpha_{n} \leq \limsup _{n \rightarrow \infty} \alpha_{n}<1$,

(ii) $0<\liminf _{n \rightarrow \infty} \beta_{n} \leq \limsup _{n \rightarrow \infty} \beta_{n}<1$.

For a given $x_{0} \in D$, define

$$
\begin{aligned}
z_{n} & =\gamma_{n} T^{n} x_{n}+\left(1-\gamma_{n}\right) x_{n}, \\
y_{n} & =\beta_{n} T^{n} z_{n}+\left(1-\beta_{n}\right) x_{n}, \\
x_{n+1} & =\alpha_{n} T^{n} y_{n}+\left(1-\alpha_{n}\right) x_{n} .
\end{aligned}
$$

Then $\left\{x_{n}\right\},\left\{y_{n}\right\}$, and $\left\{z_{n}\right\}$ converge strongly to a fixed point of $T$.

In this paper, we will extend the iterative scheme (1.1) to the iterative scheme of asymptotically quasi-nonexpansive mappings with error members. Moreover, we will prove the strong convergence of iterative scheme to a fixed point of $T$ ( $C$ need not to be a bounded set), requiring $T$ to be uniformly $L$-Lipschitzian and completely continuous. The results presented in this paper generalize and extend the corresponding main results of $\mathrm{Xu}$ and Noor [8].

\section{Preliminaries}

For the sake of convenience, we first recall some definitions and conclusions.

Definition 2.1 (see [2]). A Banach space $X$ is said to be uniformly convex if the modulus of convexity of $X$

$$
\delta_{X}(\epsilon)=\inf \left\{1-\frac{\|x+y\|}{2}:\|x\|=\|y\|=1,\|x-y\|=\epsilon\right\}>0
$$

for all $0<\epsilon \leq 2$ (i.e., $\delta_{X}(\epsilon)$ is a function $\left.(0,2] \rightarrow(0,1)\right)$.

Definition 2.2. A mapping $T: C \rightarrow C$ is called uniformly L-Lipschitzian if there exists a constant $L>0$ such that for all $x, y \in C$,

$$
\left\|T^{n} x-T^{n} y\right\| \leq L\|x-y\|, \quad \forall n \geq 1 .
$$

In what follows, we will make use of the following lemmas.

Lemma 2.3 (see [4]). Let the nonnegative number sequences $\left\{a_{n}\right\},\left\{b_{n}\right\}$, and $\left\{d_{n}\right\}$ satisfy that

$$
a_{n+1} \leq\left(1+b_{n}\right) a_{n}+d_{n}, \quad \forall n=1,2, \ldots, \sum_{n=1}^{\infty} b_{n}<\infty, \sum_{n=1}^{\infty} d_{n}<\infty
$$

Then,

(1) $\lim _{n \rightarrow \infty} a_{n}$ exists;

(2) if $\liminf _{n \rightarrow \infty} a_{n}=0$, then $\lim _{n \rightarrow \infty} a_{n}=0$. 
Lemma 2.4 ([7], J. Schu's Lemma). Let $X$ be a real uniformly convex Banach space, $0<$ $\alpha \leq t_{n} \leq \beta<1, x_{n}, y_{n} \in X, \limsup _{n \rightarrow \infty}\left\|x_{n}\right\| \leq a, \limsup _{n \rightarrow \infty}\left\|y_{n}\right\| \leq a$, and $\lim _{n \rightarrow \infty} \| t_{n} x_{n}+$ $\left(1-t_{n}\right) y_{n} \|=a, a \geq 0$. Then, $\lim _{n \rightarrow \infty}\left\|x_{n}-y_{n}\right\|=0$.

\section{Main results}

In this section, we prove our main theorem. First of all, we will need the following lemmas.

Lemma 3.1. Let $X$ be a real uniformly convex Banach space, $C$ a nonempty closed convex subset of $X$. Let $T$ be an asymptotically quasi-nonexpansive mapping with sequence $\left\{k_{n}\right\}_{n \geq 1}$ such that $\sum_{n=1}^{\infty} k_{n}<\infty$ and $F(T) \neq \varnothing$. Let $x_{0} \in C$ and

$$
\begin{aligned}
z_{n} & =\alpha_{n}^{\prime \prime} T^{n} x_{n}+\beta_{n}^{\prime \prime} x_{n}+\gamma_{n}^{\prime \prime} u_{n}, \\
y_{n} & =\alpha_{n}^{\prime} T^{n} z_{n}+\beta_{n}^{\prime} x_{n}+\gamma_{n}^{\prime} v_{n}, \\
x_{n+1} & =\alpha_{n} T^{n} y_{n}+\beta_{n} x_{n}+\gamma_{n} w_{n},
\end{aligned}
$$

where $\left\{\alpha_{n}\right\},\left\{\alpha_{n}^{\prime}\right\},\left\{\alpha_{n}^{\prime \prime}\right\},\left\{\beta_{n}\right\},\left\{\beta_{n}^{\prime}\right\},\left\{\beta_{n}^{\prime \prime}\right\},\left\{\gamma_{n}\right\},\left\{\gamma_{n}^{\prime}\right\}$, and $\left\{\gamma_{n}^{\prime \prime}\right\}$ are real sequences in $[0,1]$ and $\left\{u_{n}\right\},\left\{v_{n}\right\}$, and $\left\{w_{n}\right\}$ are three bounded sequences in $C$ such that

(i) $\alpha_{n}+\beta_{n}+\gamma_{n}=\alpha_{n}^{\prime}+\beta_{n}^{\prime}+\gamma_{n}^{\prime}=\alpha_{n}^{\prime \prime}+\beta_{n}^{\prime \prime}+\gamma_{n}^{\prime \prime}=1$,

(ii) $\sum_{n=1}^{\infty} \gamma_{n}<\infty, \sum_{n=1}^{\infty} \gamma_{n}^{\prime}<\infty, \sum_{n=1}^{\infty} \gamma_{n}^{\prime \prime}<\infty$.

If $p \in F(T)$, then $\lim _{n \rightarrow \infty}\left\|x_{n}-p\right\|$ exists.

Proof. Let $p \in F(T)$. Since $\left\{u_{n}\right\},\left\{v_{n}\right\}$, and $\left\{w_{n}\right\}$ are bounded sequences in $C$, put

$$
M=\sup _{n \geq 1}\left\|u_{n}-p\right\| \vee \sup _{n \geq 1}\left\|v_{n}-p\right\| \vee \sup _{n \geq 1}\left\|w_{n}-p\right\|
$$

Then $M$ is a finite number. So for each $n \geq 1$, we note that

$$
\begin{aligned}
\left\|x_{n+1}-p\right\| & =\left\|\alpha_{n} T^{n} y_{n}+\beta_{n} x_{n}+\gamma_{n} w_{n}-p\right\| \\
& \leq \alpha_{n}\left\|T^{n} y_{n}-p\right\|+\beta_{n}\left\|x_{n}-p\right\|+\gamma_{n}\left\|w_{n}-p\right\| \\
& \leq \alpha_{n}\left(1+k_{n}\right)\left\|y_{n}-p\right\|+\beta_{n}\left\|x_{n}-p\right\|+\gamma_{n}\left\|w_{n}-p\right\|, \\
\left\|y_{n}-p\right\| & =\left\|\alpha_{n}^{\prime} T^{n} z_{n}+\beta_{n}^{\prime} x_{n}+\gamma_{n}^{\prime} v_{n}-p\right\| \\
& \leq \alpha_{n}^{\prime}\left\|T^{n} z_{n}-p\right\|+\beta_{n}^{\prime}\left\|x_{n}-p\right\|+\gamma_{n}^{\prime}\left\|v_{n}-p\right\| \\
& \leq \alpha_{n}^{\prime}\left(1+k_{n}\right)\left\|z_{n}-p\right\|+\beta_{n}^{\prime}\left\|x_{n}-p\right\|+\gamma_{n}^{\prime}\left\|v_{n}-p\right\| \\
\left\|z_{n}-p\right\| \leq & \alpha_{n}^{\prime \prime}\left(1+k_{n}\right)\left\|x_{n}-p\right\|+\beta_{n}^{\prime \prime}\left\|x_{n}-p\right\|+\gamma_{n}^{\prime \prime}\left\|u_{n}-p\right\| .
\end{aligned}
$$




\section{Fixed points iteration}

Substituting (3.5) into (3.4),

$$
\begin{aligned}
\left\|y_{n}-p\right\| \leq & \alpha_{n}^{\prime} \alpha_{n}^{\prime \prime}\left(1+k_{n}\right)^{2}\left\|x_{n}-p\right\| \\
& +\alpha_{n}^{\prime} \beta_{n}^{\prime \prime}\left(1+k_{n}\right)\left\|x_{n}-p\right\|+\alpha_{n}^{\prime} \gamma_{n}^{\prime \prime}\left(1+k_{n}\right)\left\|u_{n}-p\right\|+\beta_{n}^{\prime}\left\|x_{n}-p\right\|+\gamma_{n}^{\prime}\left\|v_{n}-p\right\| \\
\leq & \left(1-\beta_{n}^{\prime}-\gamma_{n}^{\prime}\right) \alpha_{n}^{\prime \prime}\left(1+k_{n}\right)^{2}\left\|x_{n}-p\right\|+\beta_{n}^{\prime}\left\|x_{n}-p\right\| \\
& +\left(1-\beta_{n}^{\prime}-\gamma_{n}^{\prime}\right) \beta_{n}^{\prime \prime}\left\|x_{n}-p\right\|+m_{n} \\
\leq & \beta_{n}^{\prime}\left(1+k_{n}\right)^{2}\left\|x_{n}-p\right\|+\left(1-\beta_{n}^{\prime}\right) \alpha_{n}^{\prime \prime}\left(1+k_{n}\right)^{2}\left\|x_{n}-p\right\| \\
& +\left(1-\beta_{n}^{\prime}\right) \beta_{n}^{\prime \prime}\left(1+k_{n}\right)^{2}\left\|x_{n}-p\right\|+m_{n} \\
= & \beta_{n}^{\prime}\left(1+k_{n}\right)^{2}\left\|x_{n}-p\right\|+\left(1-\beta_{n}^{\prime}\right)\left(\alpha_{n}^{\prime \prime}+\beta_{n}^{\prime \prime}\right)\left(1+k_{n}\right)^{2}\left\|x_{n}-p\right\|+m_{n} \\
\leq & \beta_{n}^{\prime}\left(1+k_{n}\right)^{2}\left\|x_{n}-p\right\|+\left(1-\beta_{n}^{\prime}\right)\left(1+k_{n}\right)^{2}\left\|x_{n}-p\right\|+m_{n} \\
= & \left(1+k_{n}\right)^{2}\left\|x_{n}-p\right\|+m_{n},
\end{aligned}
$$

where $m_{n}=\gamma_{n}^{\prime \prime}\left(1+k_{n}\right) M+\gamma_{n}^{\prime} M$. Substituting (3.6) into (3.3) again, we have

$$
\begin{aligned}
\left\|x_{n+1}-p\right\| & \leq \alpha_{n}\left(1+k_{n}\right)\left(\left(1+k_{n}\right)^{2}\left\|x_{n}-p\right\|+m_{n}\right)+\beta_{n}\left\|x_{n}-p\right\|+\gamma_{n}\left\|w_{n}-p\right\| \\
& =\alpha_{n}\left(1+k_{n}\right)^{3}\left\|x_{n}-p\right\|+\alpha_{n}\left(1+k_{n}\right) m_{n}+\beta_{n}\left\|x_{n}-p\right\|+\gamma_{n}\left\|w_{n}-p\right\| \\
& \leq\left(\alpha_{n}+\beta_{n}\right)\left(1+k_{n}\right)^{3}\left\|x_{n}-p\right\|+\left(1+k_{n}\right) m_{n}+\gamma_{n}\left\|w_{n}-p\right\| \\
& \leq\left(1+k_{n}\right)^{3}\left\|x_{n}-p\right\|+\left(1+k_{n}\right) m_{n}+\gamma_{n}\left\|w_{n}-p\right\| \\
& \leq\left(1+k_{n}\right)^{3}\left\|x_{n}-p\right\|+\left(1+k_{n}\right) m_{n}+\gamma_{n} M \\
& =\left(1+d_{n}\right)\left\|x_{n}-p\right\|+b_{n},
\end{aligned}
$$

where $d_{n}=3 k_{n}+3 k_{n}^{2}+k_{n}^{3}$ and $b_{n}=\left(1+k_{n}\right) m_{n}+\gamma_{n} M$. Since $\sum_{n=1}^{\infty} d_{n}<\infty$ and $\sum_{n=1}^{\infty} b_{n}<$ $\infty$, by Lemma 2.3, we have that $\lim _{n \rightarrow \infty}\left\|x_{n}-p\right\|$ exists. This completes the proof.

Lemma 3.2. Let $X$ be a real uniformly convex Banach space, $C$ a nonempty closed convex subset of $X$. Let $T$ be an asymptotically quasi-nonexpansive mapping with sequence $\left\{k_{n}\right\}_{n \geq 1}$ such that $\sum_{n=1}^{\infty} k_{n}<\infty$ and $F(T) \neq \varnothing$. Let $x_{0} \in C$ and for each $n \geq 0$,

$$
\begin{aligned}
z_{n} & =\alpha_{n}^{\prime \prime} T^{n} x_{n}+\beta_{n}^{\prime \prime} x_{n}+\gamma_{n}^{\prime \prime} u_{n}, \\
y_{n} & =\alpha_{n}^{\prime} T^{n} z_{n}+\beta_{n}^{\prime} x_{n}+\gamma_{n}^{\prime} v_{n}, \\
x_{n+1} & =\alpha_{n} T^{n} y_{n}+\beta_{n} x_{n}+\gamma_{n} w_{n},
\end{aligned}
$$

where $\left\{u_{n}\right\},\left\{v_{n}\right\}$, and $\left\{w_{n}\right\}$ are three bounded sequences in $C$ and $\left\{\alpha_{n}\right\},\left\{\alpha_{n}^{\prime}\right\},\left\{\alpha_{n}^{\prime \prime}\right\},\left\{\beta_{n}\right\}$, $\left\{\beta_{n}^{\prime}\right\},\left\{\beta_{n}^{\prime \prime}\right\},\left\{\gamma_{n}\right\},\left\{\gamma_{n}^{\prime}\right\}$, and $\left\{\gamma_{n}^{\prime \prime}\right\}$ are real sequences in $[0,1]$ which satisfy the same assumptions as Lemma 3.1 and the additional assumption that $0 \leq \alpha<\alpha_{n}, \beta_{n}, \alpha_{n}^{\prime}, \beta_{n}^{\prime} \leq \beta<1$ for some $\alpha$, $\beta$ in $(0,1)$. Then $\lim _{n \rightarrow \infty}\left\|T^{n} y_{n}-x_{n}\right\|=0=\lim _{n \rightarrow \infty}\left\|T^{n} z_{n}-x_{n}\right\|$. 
Proof. For any $p \in F(T)$, it follows from Lemma 3.1, that $\lim _{n \rightarrow \infty}\left\|x_{n}-p\right\|$ exists. Let $\lim _{n \rightarrow \infty}\left\|x_{n}-p\right\|=a$ for some $a \geq 0$. From (3.6), we have

$$
\left\|y_{n}-p\right\| \leq\left(1+k_{n}\right)^{2}\left\|x_{n}-p\right\|+m_{n}
$$

Taking limsup $\operatorname{sun}_{n \rightarrow \infty}$ in both sides, we obtain

$$
\underset{n \rightarrow \infty}{\limsup }\left\|y_{n}-p\right\| \leq \limsup _{n \rightarrow \infty}\left\|x_{n}-p\right\|=\lim _{n \rightarrow \infty}\left\|x_{n}-p\right\|=a .
$$

Note that

$$
\begin{aligned}
& \limsup _{n \rightarrow \infty}\left\|T^{n} y_{n}-p\right\| \leq \limsup _{n \rightarrow \infty}\left(1+k_{n}\right)\left\|y_{n}-p\right\|=\limsup _{n \rightarrow \infty}\left\|y_{n}-p\right\| \leq a, \\
& a=\lim _{n \rightarrow \infty}\left\|x_{n+1}-p\right\|=\lim _{n \rightarrow \infty}\left\|\alpha_{n} T^{n} y_{n}+\beta_{n} x_{n}+\gamma_{n} w_{n}-p\right\| \\
& =\lim _{n \rightarrow \infty}\left\|\alpha_{n}\left[T^{n} y_{n}-p+\frac{\gamma_{n}}{2 \alpha_{n}}\left(w_{n}-p\right)\right]+\beta_{n}\left[x_{n}-p+\frac{\gamma_{n}}{2 \beta_{n}}\left(w_{n}-p\right)\right]\right\| \\
& =\lim _{n \rightarrow \infty}\left\|\alpha_{n}\left[T^{n} y_{n}-p+\frac{\gamma_{n}}{2 \alpha_{n}}\left(w_{n}-p\right)\right]+\left(1-\alpha_{n}\right)\left[x_{n}-p+\frac{\gamma_{n}}{2 \beta_{n}}\left(w_{n}-p\right)\right]\right\| .
\end{aligned}
$$

By J. Schu's Lemma 2.4, we have

$$
\lim _{n \rightarrow \infty}\left\|T^{n} y_{n}-x_{n}+\left(\frac{\gamma_{n}}{2 \alpha_{n}}-\frac{\gamma_{n}}{2 \beta_{n}}\right)\left(w_{n}-p\right)\right\|=0
$$

Since $\lim _{n \rightarrow \infty}\left\|\left(\gamma_{n} / 2 \alpha_{n}-\gamma_{n} / 2 \beta_{n}\right)\left(w_{n}-p\right)\right\|=0$, it follows that

$$
\lim _{n \rightarrow \infty}\left\|T^{n} y_{n}-x_{n}\right\|=0
$$

Finally, we will prove that $\lim _{n \rightarrow \infty}\left\|T^{n} z_{n}-x_{n}\right\|=0$. To this end, we note that for each $n \geq 1$,

$$
\left\|x_{n}-p\right\| \leq\left\|T^{n} y_{n}-x_{n}\right\|+\left\|T^{n} y_{n}-p\right\| \leq\left\|T^{n} y_{n}-x_{n}\right\|+\left(1+k_{n}\right)\left\|y_{n}-p\right\| .
$$

Since $\lim _{n \rightarrow \infty}\left\|T^{n} y_{n}-x_{n}\right\|=0=\lim _{n \rightarrow \infty} k_{n}$, we obtain that

$$
a=\lim _{n \rightarrow \infty}\left\|x_{n}-p\right\| \leq \liminf _{n \rightarrow \infty}\left\|y_{n}-p\right\|
$$

It follows that

$$
a \leq \liminf _{n \rightarrow \infty}\left\|y_{n}-p\right\| \leq \limsup _{n \rightarrow \infty}\left\|y_{n}-p\right\| \leq a .
$$

This implies that

$$
\lim _{n \rightarrow \infty}\left\|y_{n}-p\right\|=a
$$


On the other hand, we note that

$$
\begin{aligned}
\left\|z_{n}-p\right\| & =\left\|\alpha_{n}^{\prime \prime} T^{n} x_{n}+\beta_{n}^{\prime \prime} x_{n}+\gamma_{n}^{\prime \prime} u_{n}-p\right\| \\
& \leq \alpha_{n}^{\prime \prime}\left(1+k_{n}\right)\left\|x_{n}-p\right\|+\beta_{n}^{\prime \prime}\left\|x_{n}-p\right\|+\gamma_{n}^{\prime \prime}\left\|u_{n}-p\right\| \\
& \leq \alpha_{n}^{\prime \prime}\left(1+k_{n}\right)\left\|x_{n}-p\right\|+\left(1-\alpha_{n}^{\prime \prime}\right)\left(1+k_{n}\right)\left\|x_{n}-p\right\|+\gamma_{n}^{\prime \prime}\left\|u_{n}-p\right\| \\
& \leq\left(1+k_{n}\right)\left\|x_{n}-p\right\|+\gamma_{n}^{\prime \prime}\left\|u_{n}-p\right\| .
\end{aligned}
$$

By boundedness of the sequence $\left\{u_{n}\right\}$ and $\lim _{n \rightarrow \infty} k_{n}=0=\lim _{n \rightarrow \infty} \gamma_{n}^{\prime \prime}$, we have

$$
\underset{n \rightarrow \infty}{\limsup }\left\|z_{n}-p\right\| \leq \limsup _{n \rightarrow \infty}\left\|x_{n}-p\right\|=a,
$$

and so

$$
\begin{gathered}
\limsup _{n \rightarrow \infty}\left\|T^{n} z_{n}-p\right\| \leq \limsup _{n \rightarrow \infty}\left(1+k_{n}\right)\left\|z_{n}-p\right\| \leq a, \\
a=\lim _{n \rightarrow \infty}\left\|y_{n}-p\right\|=\lim _{n \rightarrow \infty}\left\|\alpha_{n}^{\prime} T^{n} z_{n}+\beta_{n}^{\prime} x_{n}+\gamma_{n}^{\prime} v_{n}-p\right\| \\
=\lim _{n \rightarrow \infty}\left\|\alpha_{n}^{\prime}\left[T^{n} z_{n}-p+\frac{\gamma_{n}^{\prime}}{2 \alpha_{n}^{\prime}}\left(v_{n}-p\right)\right]+\beta_{n}^{\prime}\left[x_{n}-p+\frac{\gamma_{n}^{\prime}}{2 \beta_{n}^{\prime}}\left(v_{n}-p\right)\right]\right\| \\
=\lim _{n \rightarrow \infty}\left\|\alpha_{n}^{\prime}\left[T^{n} z_{n}-p+\frac{\gamma_{n}^{\prime}}{2 \alpha_{n}^{\prime}}\left(v_{n}-p\right)\right]+\left(1-\alpha_{n}^{\prime}\right)\left[x_{n}-p+\frac{\gamma_{n}^{\prime}}{2 \beta_{n}^{\prime}}\left(v_{n}-p\right)\right]\right\| .
\end{gathered}
$$

By J. Schu's Lemma 2.4, we have

$$
\lim _{n \rightarrow \infty}\left\|T^{n} z_{n}-x_{n}+\left(\frac{\gamma_{n}^{\prime}}{2 \alpha_{n}^{\prime}}-\frac{\gamma_{n}^{\prime}}{2 \beta_{n}^{\prime}}\right)\left(v_{n}-p\right)\right\|=0
$$

Since $\lim _{n \rightarrow \infty}\left\|\left(\gamma_{n}^{\prime} / 2 \alpha_{n}^{\prime}-\gamma_{n}^{\prime} / 2 \beta_{n}^{\prime}\right)\left(v_{n}-p\right)\right\|=0$, it follows that

$$
\lim _{n \rightarrow \infty}\left\|T^{n} z_{n}-x_{n}\right\|=0 .
$$

This completes the proof.

Theorem 3.3. Let $X$ be a real uniformly convex Banach space, $C$ a nonempty closed convex subset of $X$. Let $T$ be uniformly L-Lipschitzian, completely continuous, and an asymptotically quasi-nonexpansive mapping with sequence $\left\{k_{n}\right\}_{n \geq 1}$ such that $\sum_{n=1}^{\infty} k_{n}<\infty$ and $F(T) \neq \varnothing$. Let $x_{0} \in C$ and for each $n \geq 0$,

$$
\begin{aligned}
z_{n} & =\alpha_{n}^{\prime \prime} T^{n} x_{n}+\beta_{n}^{\prime \prime} x_{n}+\gamma_{n}^{\prime \prime} u_{n}, \\
y_{n} & =\alpha_{n}^{\prime} T^{n} z_{n}+\beta_{n}^{\prime} x_{n}+\gamma_{n}^{\prime} v_{n}, \\
x_{n+1} & =\alpha_{n} T^{n} y_{n}+\beta_{n} x_{n}+\gamma_{n} w_{n},
\end{aligned}
$$

where $\left\{u_{n}\right\},\left\{v_{n}\right\}$, and $\left\{w_{n}\right\}$ are three bounded sequences in $C$ and $\left\{\alpha_{n}\right\},\left\{\alpha_{n}^{\prime}\right\},\left\{\alpha_{n}^{\prime \prime}\right\},\left\{\beta_{n}\right\}$, $\left\{\beta_{n}^{\prime}\right\},\left\{\beta_{n}^{\prime \prime}\right\},\left\{\gamma_{n}\right\},\left\{\gamma_{n}^{\prime}\right\}$, and $\left\{\gamma_{n}^{\prime \prime}\right\}$ are real sequences in $[0,1]$ which satisfy the same assumptions as Lemma 3.1 and the additional assumption that $0 \leq \alpha<\alpha_{n}, \beta_{n}, \alpha_{n}^{\prime}, \beta_{n}^{\prime} \leq \beta<1$ for some $\alpha, \beta$ in $(0,1)$. Then $\left\{x_{n}\right\},\left\{y_{n}\right\}$, and $\left\{z_{n}\right\}$ converge strongly to a fixed point of $T$. 
Proof. It follows from Lemma 3.2 that

$$
\lim _{n \rightarrow \infty}\left\|T^{n} y_{n}-x_{n}\right\|=0=\lim _{n \rightarrow \infty}\left\|T^{n} z_{n}-x_{n}\right\|
$$

and this implies that

$$
\left\|x_{n+1}-x_{n}\right\| \leq \alpha_{n}\left\|T^{n} y_{n}-x_{n}\right\|+y_{n}\left\|w_{n}-x_{n}\right\| \longrightarrow 0 \quad \text { as } n \longrightarrow \infty .
$$

We note that

$$
\begin{aligned}
\left\|T^{n} x_{n}-x_{n}\right\| & \leq\left\|T^{n} x_{n}-T^{n} y_{n}\right\|+\left\|T^{n} y_{n}-x_{n}\right\| \leq L\left\|x_{n}-y_{n}\right\|+\left\|T^{n} y_{n}-x_{n}\right\| \\
& \leq \alpha_{n}^{\prime} L\left\|x_{n}-T^{n} z_{n}\right\|+\gamma_{n}^{\prime} L\left\|x_{n}-x_{n}\right\|+\left\|T^{n} y_{n}-x_{n}\right\| \rightarrow 0 \quad \text { as } n \longrightarrow \infty \\
\left\|x_{n}-T x_{n}\right\| & \leq\left\|x_{n+1}-x_{n}\right\|+\left\|x_{n+1}-T^{n+1} x_{n+1}\right\|+\left\|T^{n+1} x_{n+1}-T^{n+1} x_{n}\right\|+\left\|T^{n+1} x_{n}-T x_{n}\right\| \\
& \leq\left\|x_{n+1}-x_{n}\right\|+\left\|x_{n+1}-T^{n+1} x_{n+1}\right\|+\left(1+k_{n+1}\right)\left\|x_{n+1}-x_{n}\right\|+L\left\|T^{n} x_{n}-x_{n}\right\| .
\end{aligned}
$$

It follows from (3.25), (3.26), and the above inequality that

$$
\lim _{n \rightarrow \infty}\left\|x_{n}-T x_{n}\right\|=0
$$

By Lemma 3.1, $\left\{x_{n}\right\}$ is bounded. It follows from our assumption that $T$ is completely continuous and that there exists a subsequence $\left\{T x_{n_{k}}\right\}$ of $\left\{T x_{n}\right\}$ such that $T x_{n_{k}} \rightarrow p \in C$ as $k \rightarrow \infty$. Moreover, by (3.28), we have $\left\|T x_{n_{k}}-x_{n_{k}}\right\| \rightarrow 0$ which implies that $x_{n_{k}} \rightarrow p$ as $k \rightarrow \infty$. By (3.28) again, we have

$$
\|p-T p\|=\lim _{k \rightarrow \infty}\left\|x_{n_{k}}-T x_{n_{k}}\right\|=0 .
$$

This shows that $p \in F(T)$. Furthermore, since $\lim _{n \rightarrow \infty}\left\|x_{n}-p\right\|$ exists, we have $\lim _{n \rightarrow \infty}\left\|x_{n}-p\right\|=0$, that is, $\left\{x_{n}\right\}$ converges to some fixed point of $T$. It follows that

$$
\begin{aligned}
& \left\|y_{n}-x_{n}\right\| \leq \alpha_{n}^{\prime}\left\|T^{n} z_{n}-x_{n}\right\|+\gamma_{n}^{\prime}\left\|v_{n}-x_{n}\right\| \longrightarrow 0, \\
& \left\|z_{n}-x_{n}\right\| \leq \alpha_{n}^{\prime \prime}\left\|T^{n} x_{n}-x_{n}\right\|+\gamma_{n}^{\prime \prime}\left\|u_{n}-x_{n}\right\| \longrightarrow 0
\end{aligned}
$$

Therefore, $\lim _{n \rightarrow \infty} y_{n}=p=\lim _{n \rightarrow \infty} z_{n}$. This completes the proof.

\section{References}

[1] R. Glowinski and P. Le Tallec, Augmented Lagrangian and Operator-Splitting Methods in Nonlinear Mechanics, SIAM Studies in Applied Mathematics, vol. 9, SIAM, Pennsylvania, 1989.

[2] K. Goebel and W. A. Kirk, A fixed point theorem for asymptotically nonexpansive mappings, Proc. Amer. Math. Soc. 35 (1972), 171-174.

[3] S. Haubruge, V. H. Nguyen, and J. J. Strodiot, Convergence analysis and applications of the Glowinski-Le Tallec splitting method for finding a zero of the sum of two maximal monotone operators, J. Optim. Theory Appl. 97 (1998), no. 3, 645-673. 


\section{Fixed points iteration}

[4] Q. Liu, Iteration sequences for asymptotically quasi-nonexpansive mapping with an error member of uniform convex Banach space, J. Math. Anal. Appl. 266 (2002), no. 2, 468-471.

[5] M. A. Noor, New approximation schemes for general variational inequalities, J. Math. Anal. Appl. 251 (2000), no. 1, 217-229.

[6] Three-step iterative algorithms for multivalued quasi variational inclusions, J. Math. Anal. Appl. 255 (2001), no. 2, 589-604.

[7] J. Schu, Iterative construction of fixed points of strictly pseudocontractive mappings, Appl. Anal. 40 (1991), no. 2-3, 67-72.

[8] B. Xu and M. A. Noor, Fixed-point iterations for asymptotically nonexpansive mappings in Banach spaces, J. Math. Anal. Appl. 267 (2002), no. 2, 444-453.

Somyot Plubtieng: Department of Mathematics, Faculty of Science, Naresuan University, Phitsanulok 65000, Thailand

E-mail address: somyotp@nu.ac.th

Rabian Wangkeeree: Department of Mathematics, Faculty of Science, Naresuan University, Phitsanulok 65000, Thailand

E-mail address: rabianw@nu.ac.th 


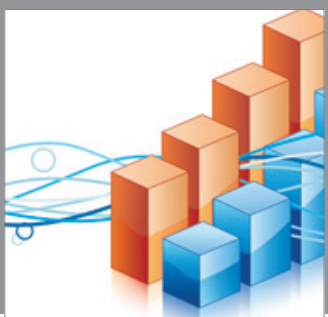

Advances in

Operations Research

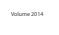

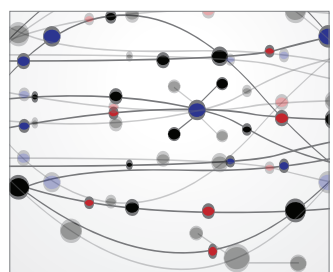

\section{The Scientific} World Journal
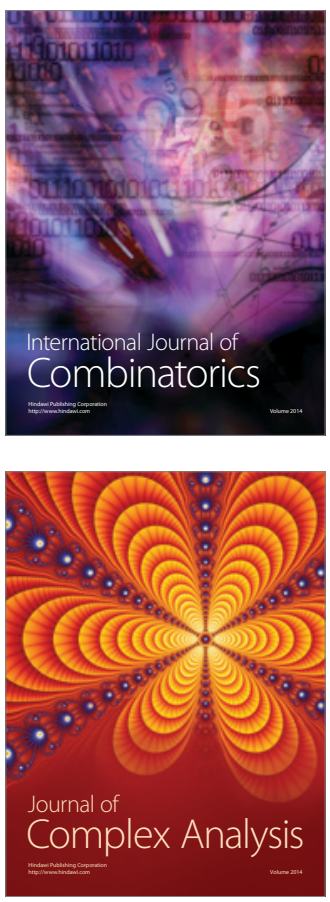

International Journal of

Mathematics and

Mathematical

Sciences
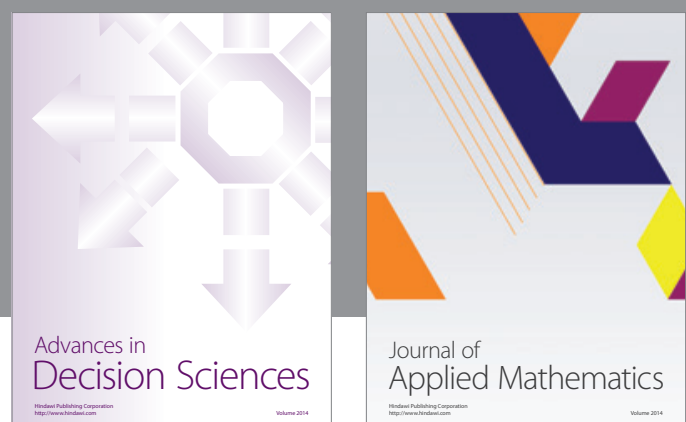

Journal of

Applied Mathematics
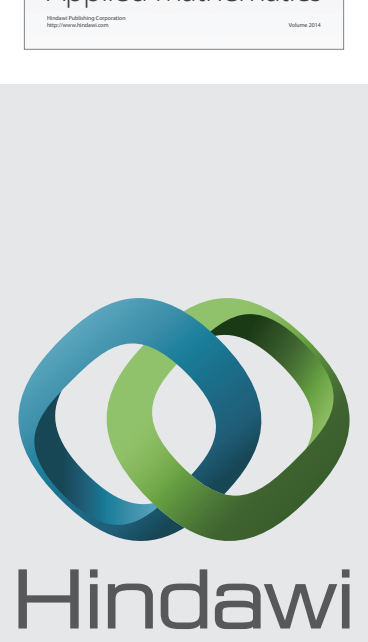

Submit your manuscripts at http://www.hindawi.com
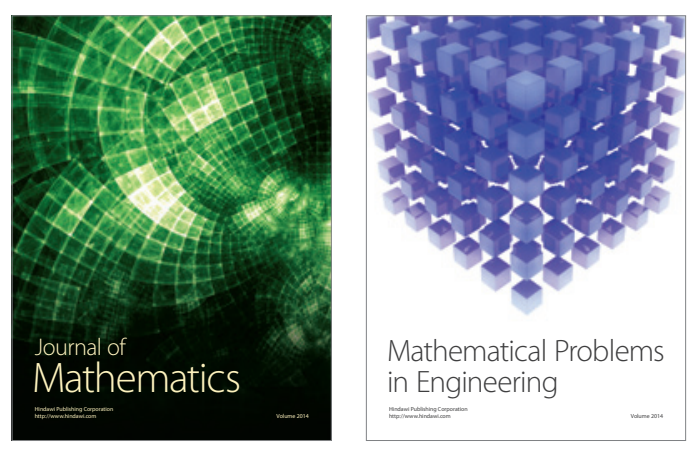

Mathematical Problems in Engineering
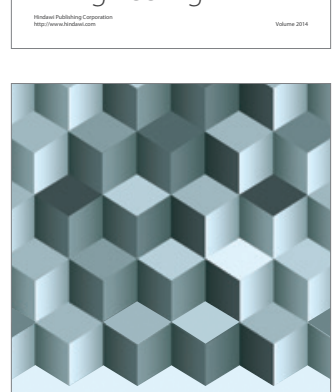

Journal of

Function Spaces
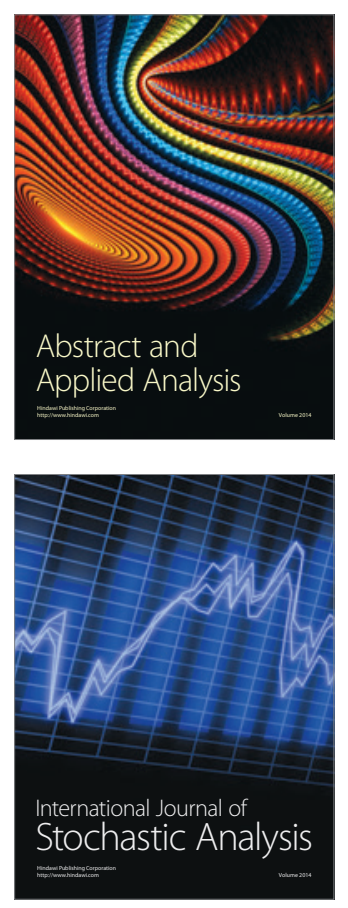

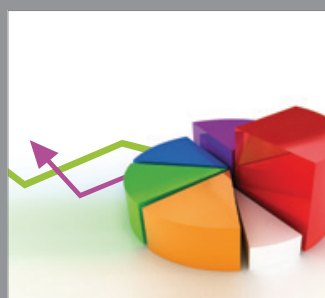

ournal of

Probability and Statistics

Promensencen
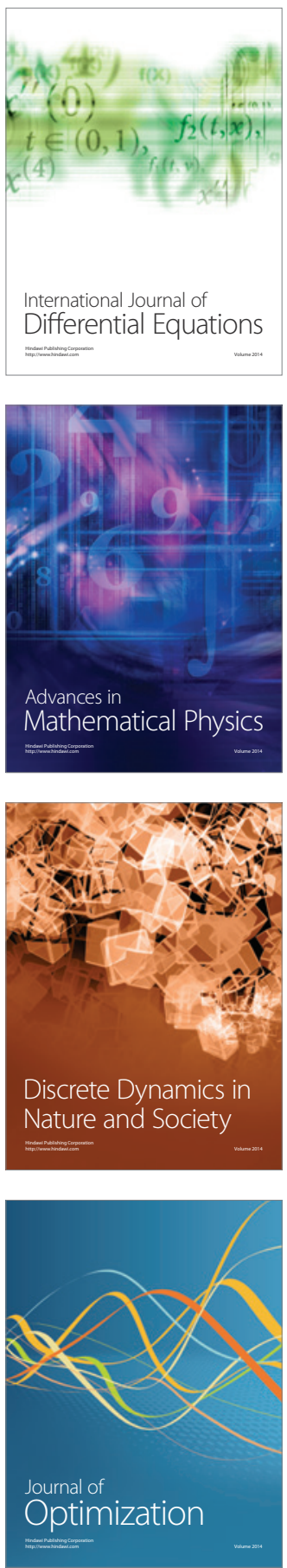